\title{
Finding an alternative diagnosis does not justify increased use of CT-pulmonary angiography
}

\author{
Subani Chandra', Pralay K Sarkar ${ }^{1}$, Divay Chandra ${ }^{2}$, Nicole E Ginsberg ${ }^{1}$ and Rubin I Cohen ${ }^{1 *}$
}

\begin{abstract}
Background: The increased use of computed tomography pulmonary angiography (CTPA) is often justified by finding alternative diagnoses explaining patients' symptoms. However, this has not been rigorously examined.

Methods: We retrospectively reviewed CTPA done at our center over an eleven year period (2000 - 2010) in patients with suspected pulmonary embolus (PE). We then reviewed in detail the medical records of a representative sample of patients in three index years - 2000, 2005 and 2008. We determined whether CTPA revealed pulmonary pathology other than PE that was not readily identifiable from the patient's history, physical examination and prior chest X-ray. We also assessed whether the use of pre-test probability guided diagnostic strategy for PE.

Results: A total of 12,640 CTPA were performed at our center from year 2000 to 2010. The number of CTPA performed increased from 84 in 2000 to 2287 in 2010, a 27 fold increase. Only 7.6 percent of all CTPA and 3.2 percent of avoidable CTPAs (low or intermediate pre-test probability and negative D-dimer) revealed previously unknown findings of any clinical significance. When we compared 2008 to 2000 and 2005, more CTPAs were performed in younger patients (mean age (years) for 2000: 67, 2005: 63, and 2008: 60, ( $p=0.004$, one-way ANOVA)). Patients were less acutely ill with fewer risk factors for PE. Assessment of pre-test probability of PE and D-dimer measurement were rarely used to select appropriate patients for CTPA (pre-test probability of PE documented in chart (\% total) in year 2000: 4.1\%, 2005: 1.6\%, 2008: 3.1\%).
\end{abstract}

Conclusions: Our data do not support the argument that increased CTPA use is justified by finding an alternative pulmonary pathology that could explain patients' symptoms. CTPA is being increasingly used as the first and only test for suspected PE.

\section{Background}

Computed tomography pulmonary angiography (CTPA) is the preferred method to confirm or exclude a PE. However the non-selective use of CTPA has several disadvantages. These include long term risks of exposure to high doses of radiation and a small but definite risk of kidney injury due to intravenous contrast. Moreover, CTPA is an expensive test and often leaves behind a trail of incidental findings of indeterminate implication that further increase health care costs [1,2]. Even with CTPA's technological advances, easy availability and ever shorter scan times, the assessment of clinical pre-test

\footnotetext{
* Correspondence: rocohen@nshs.edu

'Division of Pulmonary, Critical Care and Sleep Medicine, The Long Island Jewish Medical Center, The Hofstra North Shore-LIJ School of Medicine, New Hyde Park, New York, NY 11040, USA

Full list of author information is available at the end of the article
}

probability remains central to the diagnosis of PE and ideally should influence the initial choice of diagnostic testing [3-5]. However, it remains unclear how frequently pre-test probability algorithms are followed in daily clinical practice [6-8]. Furthermore, while the number of CTPA has increased dramatically, the majority of CTPA do not show the presence of PE. Nevertheless, the increase in the use of CTPA is often justified by the discovery of hitherto unknown pathological etiologies [9-13]; however, review of the literature would indicate that this has not been rigorously examined. In this study we examined the patterns of CTPA use over an eleven year period at a single center and assessed whether the use of CTPA was justified in those with low or intermediate pre-test probability by the finding of an alternative diagnosis that might have explained the patient's symptoms. 


\section{Methods}

We determined the total number of CTPA performed in patients $>18$ years in age, and their results (as either positive or negative for PE) for each year from 20002010 in a 500 bed, academic teaching hospital in New York City. The study was approved by the North ShoreLong Island Jewish Health System's institutional review board which waived the need for informed consent.

We studied in detail, the medical records of patients who underwent CTPA in three index years 2000, 2005, and 2008. We chose the year 2000 as a baseline reference year, the year 2005 was the first calendar year after the hospital obtained an additional CT scanner, and the year 2008 followed the publication of Prospective Investigation of Pulmonary Embolism Diagnosis II (PIOPED II) and its recommendations [3-5,14]. We reviewed all available records for the year 2000 (74 records) and a random sample representative of all CTPA performed in 2005 and 2008. A total of 850 studies were reviewed for 2005 and 2008. We excluded CT chest angiograms performed for other reasons such as aortic dissection. Demographic data, clinical presentation, risk factors for thromboembolic disease, documentation of pre-test probability of $\mathrm{PE}$, use of therapeutic anticoagulation, and results of CTPA were abstracted from patients' medical records. Since increased use of CTPA may be replacing that of $\mathrm{V} / \mathrm{Q}$ scans, we also determined the number of $\mathrm{V} / \mathrm{Q}$ scans performed for the diagnosis of $\mathrm{PE}$ from 2000 to 2010.

\section{Assessment of pre-test probability of PE}

We assigned pre-test probability to all subjects using the Revised Geneva Score (RGS) [15]. At the time of assignment of pre-test probability, the investigators were blinded to the results of the CTPA. Based on the RGS we assigned subjects to low (RGS 0-3), intermediate (RGS $4-10$ ), and high (RGS $\geq 11$ ) pre-test probability categories. CTPA performed on patients who were in the low or intermediate pre-test probability category and had a negative D-dimer were considered avoidable.

\section{D-dimer measurement}

In 2000 and 2005, D-dimer was measured by quantitative latex agglutination and in 2008 by ELISA (Enzymelinked immunosorbent assay) with fluorescence (ELFA) (VIDAS ${ }^{\circledR}$ D-dimer Exclusion TM, bioMérieux, Marcy l'Etoile, France). The D-dimer assay is readily available in our hospital and the results are reported within 30 minutes.

\section{CT pulmonary angiography}

In 2000, CTPAs were performed on single or 4 - slice scanners (HiSpeed, GE Healthcare, UK). In 2005 another 4-slice CT scanner (HiSpeed, GE Healthcare, UK) was installed and the existing CT scanners were upgraded to 16-slice CT machines (LightSpeed, GE Healthcare, UK). In 2008, all CTPA were being performed on 16-slice scanners (LightSpeed and BrightSpeed, GE Healthcare, UK). Central PE was defined as PE in the pulmonary trunk, right or left main pulmonary arteries or lobar arteries while PE in segmental or sub-segmental branches were considered peripheral.

\section{Sample size calculation}

We used an estimate of the proportion of patients who had a CTPA and were in the low probability group for the sample size calculations. Statistically, the most conservative calculation occurs if $50 \%$ of patients fall into this category. We imposed a constraint to estimating within 5 percentage points of the expected proportion since the sample size for the years 2005 and 2008 was so large. For a sample size of 385, a two-sided 95\% confidence interval for a single proportion is no wider than \pm $5.0 \%$ from an expected overall proportion of $50 \%$ (i.e. $45 \%$ to $55 \%$ ). Based on this estimate a random sample of 383 and 393 CTPA were reviewed for 2005 and 2008 respectively. We reviewed all available CTPA results (74 of 84) for the year 2000.

\section{Statistical analyses}

All analyses were performed using Stata 11.1 (StataCorp LP, College Station, TX). Chi-square tests were used for categorical data, while one-way ANOVA was used for continuous variables. A two-tailed $p$-value $<0.05$ was considered statistically significant.

\section{Results}

Over the study period (2000 to 2010), there was a 27fold increase in the total number of CTPA (Figure 1) without a corresponding increase in yield. The total number of CTPA in the three index years 2000, 2005 and 2008 were 84,1114 and 2287 respectively. While the number of CTPA ordered increased in all departments, the ED had the largest increase. The numbers of scans performed in the ED per 100 visits were 0.14 in 2000, 1.82 in 2005 and 2.58 in 2008. The ED also had the steepest decline in the percentage of scans positive for PE (Figure 2).

\section{Justification for CTPA}

We assessed whether the use of CTPA is justified in those with low or intermediate pre-test probability by the finding of an alternative diagnosis that might explain the patient's symptoms. The frequency of alternate diagnoses found on CTPA was as follows: ED: 10\%, Medicine: $5 \%$, Surgery: $3 \% ; p=0.08$. When we examine the data more closely, only $3.2 \%$ of potentially avoidable CTPA (low or intermediate pre-test probability and 


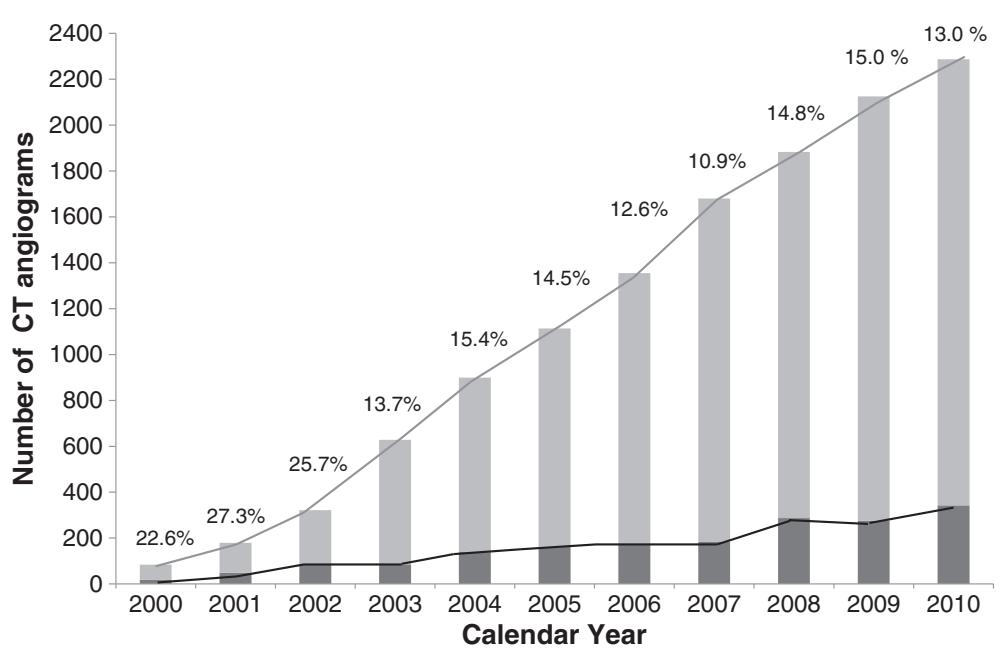

Figure 1 The number of CTPA performed per calendar year from 2000 to $\mathbf{2 0 1 1}$. The dark bars and percentages are the CTPA that were positive for PE.

negative D-dimer) had an alternative diagnosis that was neither previously known nor evident on a chest radiograph performed prior to the CTPA.

\section{Findings on prior chest X-ray}

$13 \%$ of the patients did not have a prior chest X-ray defined as within 48 hours of CTPA. Among those who did undergo a chest X-ray, the findings were as follows: $40 \%$ normal, $14 \%$ pleural effusion, $11 \%$ pulmonary edema, $6 \%$ atelectasis, $6 \%$ infiltrate, and $10 \%$ other.

\section{Patients undergoing CTPA}

In contrast to 2000 and 2005, those undergoing CTPA in 2008 were significantly younger and less acutely ill as evidenced by their higher oxygen saturation and lower respiratory rate (Table 1). Moreover, patients in 2008 were much less likely to have any risk factors for thrombosis or a prior history of venous thromboembolism. There was an increase in the use of CTPA in patients with chest pain, and for "other" indications (Table 1). The increase in number of scans ordered was accompanied by a significant drop in the diagnosis of PE from $22.6 \%$ in 2000 to $13 \%$ in 2010 . We also noted a change in the size and location of pulmonary emboli detected over time: in 2008, a greater proportion of PE was found distal to the lobar arteries in the segmental or sub segmental branches (Table 2). Since younger patients and women may be more at risk from the potential carcinogenic

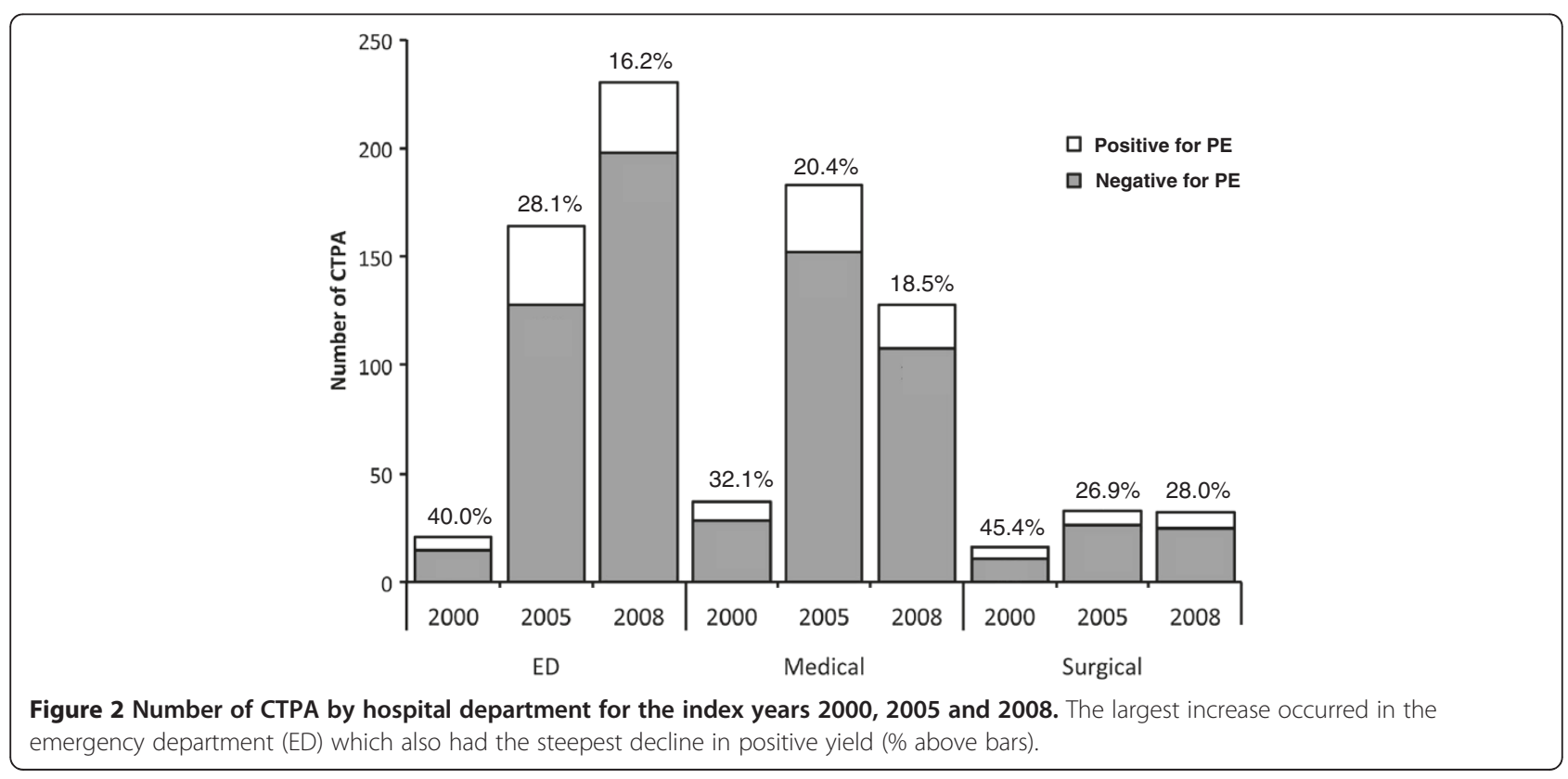


Table 1 Demographics, signs and symptoms, risk factors and prior medical history by year for 850 patients undergoing CT angiography

\begin{tabular}{|c|c|c|c|c|c|}
\hline Characteristics & 2000 & 2005 & 2008 & Total & $\mathbf{p}$ \\
\hline$n$ & 74 & 383 & 393 & 850 & \\
\hline \multicolumn{6}{|l|}{ Demographics } \\
\hline Age (years) (mean \pm SD) & $66.8 \pm 16.8$ & $62.9 \pm 17$ & $60 \pm 19.3$ & $61.9 \pm 18.2$ & 0.004 \\
\hline Female (\%) & 59.5 & 65 & 67.9 & 65.9 & 0.33 \\
\hline Females $<40$ years $(\%)$ & 6.8 & 7.8 & 16.5 & 11.8 & 0.004 \\
\hline Body mass index $\left(\mathrm{kg} / \mathrm{m}^{2}\right)($ mean $\pm \mathrm{SD})$ & $27.3 \pm 5.5$ & $27.4 \pm 7.2$ & $27.8 \pm 6.2$ & $27.6 \pm 6.7$ & 0.760 \\
\hline
\end{tabular}

\section{Signs and symptoms}

Shortness of breath (\%)

Chest pain (\%)

Palpitations (\%)

Syncope (\%)

Hypoxia (\%)

Cough (\%)

Hemoptysis (\%)

Lower extremity pain (\%)

Leg edema/tenderness (\%)

Other (\%)

Systolic blood pressure $(\mathrm{mmHg})$

Diastolic blood pressure $(\mathrm{mmHg})$

Respiratory rate (/min)

Oxygen saturation (\%)

Requiring supplemental O2 (\%)

Risk factors for thrombosis (\%)

Immobility

Active malignancy

Hospitalized in the prior 4 weeks

Surgery in the prior 4 weeks

Prior PE or DVT

Pregnant

Known hypercoagulable disorder

On estrogen therapy

\section{Prior medical history (\%)}

Any lung disease

Hypertension

Coronary artery disease

Congestive heart failure

Other cardiac disease

Dialysis

Transferred from another institution

\begin{tabular}{|c|c|c|c|c|}
\hline 79.7 & 63.7 & 62.3 & 64.5 & 0.015 \\
\hline 27 & 38.9 & 50.1 & 43.1 & $<0.001$ \\
\hline 5.5 & 1.3 & 3.3 & 2.6 & 0.06 \\
\hline 2.7 & 1 & 3.8 & 2.5 & 0.04 \\
\hline 13.7 & 6.5 & 10.2 & 8.8 & 0.06 \\
\hline 9.5 & 9.9 & 12 & 10.8 & 0.60 \\
\hline 1.4 & 0.3 & 3.6 & 1.9 & 0.003 \\
\hline 4.1 & 0 & 5.9 & 3.1 & $<0.001$ \\
\hline 1.4 & 0 & 8.9 & 4.2 & $<0.001$ \\
\hline 14.9 & 10.2 & 27.2 & 18.5 & $<0.001$ \\
\hline $128 \pm 27$ & $131 \pm 23$ & $129 \pm 22$ & $130 \pm 23$ & 0.389 \\
\hline $73 \pm 13$ & $75 \pm 15$ & $73 \pm 14$ & $74 \pm 14$ & 0.19 \\
\hline $23.8 \pm 7.5$ & $20.7 \pm 6$ & $20 \pm 5.7$ & $20.6 \pm 6$ & $<0.001$ \\
\hline $92.7 \pm 6.8$ & $94.1 \pm 6$ & $95.3 \pm 9.6$ & $94.5 \pm 8$ & 0.01 \\
\hline 40.5 & 22.1 & 19 & 22.3 & $<0.001$ \\
\hline 31.1 & 24.8 & 15.8 & 21.2 & 0.001 \\
\hline 31.1 & 29.8 & 22.1 & 26.4 & 0.03 \\
\hline 38.4 & 34.7 & 28.3 & 32.1 & 0.08 \\
\hline 25.7 & 17 & 16.3 & 17.4 & 0.14 \\
\hline 17.6 & 9.4 & 12.5 & 11.5 & 0.01 \\
\hline 1.4 & 1.6 & 5.1 & 3.2 & 0.01 \\
\hline 0 & 1.6 & 1 & 1.2 & 0.48 \\
\hline 6.8 & 1.8 & 3.6 & 3.1 & 0.06 \\
\hline 29.7 & 26.4 & 24.4 & 25.8 & 0.58 \\
\hline 48.7 & 16.5 & 51.2 & 35.3 & $<0.001$ \\
\hline 27 & 18.5 & 23.2 & 21.4 & 0.14 \\
\hline 18.9 & 6 & 6.6 & 7.4 & $<0.001$ \\
\hline 25.8 & 6.8 & 13.5 & 11.5 & 0.26 \\
\hline 0 & 1.3 & 2.3 & 1.7 & 0.28 \\
\hline 8.2 & 11.5 & 8.4 & 9.8 & 0.31 \\
\hline
\end{tabular}


Table 2 CTPA positivity rate and location of pulmonary emboli

\begin{tabular}{lccccc}
\hline CTPA Results & $\mathbf{2 0 0 0}$ & $\mathbf{2 0 0 5}$ & $\mathbf{2 0 0 8}$ & Total & $\mathbf{p}$ \\
\hline $\boldsymbol{n}$ & 74 & 383 & 393 & 850 & \\
$\begin{array}{l}\text { Embolus present (\%) } \\
\begin{array}{l}\text { Location of embolus } \\
\text { for positive studies (\%) }\end{array}\end{array}$ & 25.7 & 19.1 & 14.8 & 17.7 & $\mathbf{0 . 0 4 8}$ \\
$\quad \begin{array}{l}\text { Central PE } \\
\text { Peripheral PE }\end{array}$ & 89.6 & 46.6 & 51.8 & 53.9 & $\mathbf{0 . 0 0 3}$ \\
& 10.5 & 53.4 & 48.3 & 46 &
\end{tabular}

Central PE was defined as PE in the pulmonary trunk, right or left main pulmonary arteries or lobar arteries while PE in segmental or sub-segmental branches were considered peripheral.

There was no correlation between pre-test probability by RGS and location or size of $\mathrm{PE}$.

effects of ionizing radiation [16], we also examined age and gender in our sample. The number of CTPA performed on women under the age of 40 years increased significantly over the study period (Table 1). While $11.8 \%$ of all CTPAs performed were on women less than 40 years of age, the presence of a PE in this cohort was lower than in all CTPAs reviewed (9.1\% versus $17.7 \%$ ).

\section{Pre-test probability}

Across all years, physicians ordering CTPA rarely documented the pre-test probability of PE in the patients' medical records (Table 3). Our calculation of pre-test probability based on the RGS showed that more patients with low pre-test probability of PE underwent CTPA in later years (Table 3). Overall, PE was present on CTPA in $9.3 \%$ of patients with a low pre-test probability, $20.9 \%$ patients with an intermediate pre-test probability and $29.6 \%$ with a high pre-test probability of PE. Slightly over one fifth $(22.2 \%)$ of patients with a high pre-test probability of PE received any form of anticoagulation prior to undergoing CTPA.

\section{Use of D-dimer}

D-dimer was assessed in an increasing number of patients with low or intermediate pre-test probability of PE. In 2000, no patient with a low or intermediate RGS underwent a D-dimer test. In 2005 this number was $21.3 \%$ and in 2008 was $31.9 \%$. Notably, our data show that when the D-dimer was negative in such patients and PE was not diagnosed on CTPA (Table 4). Among those with high pre-test probability, the D-dimer was always positive when performed.

\section{V/Q scanning}

Since the increase in CTPA may be mirrored by a corresponding decrease in V/Q scans, we were interested as to whether the number of V/Q performed for the diagnosis of PE decreased accordingly (Figure 3). The installation and availability of another CT scanner in 2005 resulted in 19\% drop in V/Q scans compared to 2000. By 2008, the number of V/Q scans being performed for the diagnosis of PE dropped by 31\% compared to 2005 (Table 5), and by $44 \%$ compared to 2000 . Table 5 also illustrates the changes in ED visits and hospital admissions for the index years 2000, 2005 and 2008.

\section{Discussion}

From the year 2000 to 2010, the total number of CTPA performed at our center increased exponentially although the percentage of scans positive for PE declined. This increase in CTPA was not accompanied (or justified) by finding an alternative diagnoses on CTPA that explains the patients' symptoms. Indeed, our data indicate that CTPA rarely reveals a previously unknown or new finding especially in those with low and intermediate risk for PE. CTPA provided an alternate diagnosis, defined as one that was not previously known or evident on chest radiograph in only $7.6 \%$ of all non-PE CTPA. Additionally, this number would drop to $3.2 \%$ had the published guidelines for investigation of suspected PE been followed, that is in those with low or intermediate pre-test probability and a negative $\mathrm{D}$ dimer. We also find that CTPAs were being performed in younger patients even in the absence of symptoms and identifiable PE risk factors further negating the argument of discovering an alternative pathological diagnosis. Finally, the increase in the number of CTPA corresponds neither to the decrease in the number of $\mathrm{V} / \mathrm{Q}$ scans nor to the increase in the number of ED visits or hospital admissions.

The utility of CTPA to provide an alternative diagnosis is often cited as justification for obtaining this test even

Table 3 Assessment of clinical pre-test probability and RGS

\begin{tabular}{|c|c|c|c|c|c|}
\hline & 2000 & 2005 & 2008 & Total & $\mathbf{p}$ \\
\hline$n$ & 74 & 383 & 393 & 850 & \\
\hline Pre-test probability of PE documented (\%) & 4.1 & 1.6 & 3.1 & 2.4 & 0.48 \\
\hline Calculated Revised Geneva Score (mean $\pm S D$ ) & $5.7 \pm 2.9$ & $5.3 \pm 2.5$ & $5.4 \pm 3.0$ & $5.4 \pm 2.8$ & 0.42 \\
\hline Low probability (\%) & 25.7 & 24.5 & 28.8 & 26.6 & \\
\hline Intermediate probability (\%) & 70.3 & 74.4 & 66.2 & 70.2 & 0.01 \\
\hline High probability (\%) & 4.1 & 1 & 5.1 & 3.2 & \\
\hline
\end{tabular}


Table 4 Use of D-dimer in patients undergoing CTPA

\begin{tabular}{lcccc}
\hline $\begin{array}{l}\text { Pre-test } \\
\text { probability }\end{array}$ & $\mathbf{n}$ & $\begin{array}{c}\text { D-dimer not performed, \% } \\
\text { (CTPA positive \%) }\end{array}$ & Negative D-dimer, \% (CTPA positive, \%) & Positive D-dimer, \% (CTPA positive, \%) \\
\hline Low & & & & D-dimer performed \\
2000 & 19 & $100.0(100)$ & $4.3(0)$ & $17(0)$ \\
2005 & 94 & $78.7(6.8)$ & $7(0)$ & $24.8(7.1)$ \\
2008 & 113 & $68.1(16.9)$ & & - \\
Intermediate & & & $1.9(0)$ & $17.2(24.5)$ \\
2000 & 52 & $98.1(33.3)$ & $1.8(0)$ & $16.2(21.4)$ \\
2005 & 285 & $81.0(23.8)$ & $4.6(0)$ & - \\
2008 & 260 & $79.2(15.5)$ & & $25(100)$ \\
High & & & - & $15(0)$ \\
2000 & 3 & $100.0(66.7)$ & - & - \\
2005 & 4 & $75.0(33.3)$ & $85.0(23.5)$ &
\end{tabular}

when PE is not found [9-15]. In prior studies, the most frequently cited alternative diagnosis on CTPA is an infiltrate or consolidation suggestive of pneumonia. However, it is not recorded whether a history and physical examination had already suggested pneumonia; neither was it noted whether a chest radiogram was performed prior to the CTPA. While it is difficult to compare studies as purpose, setting and design differ, one retrospective study reported a third of CTPAs revealed an alternative explanation of the patient's presenting symptoms but such findings were already known in over half of patients from the admission chest radiogram [9,17]. In our center, $40 \%$ of the chest X-rays performed prior to the CTPA were interpreted as normal, while in $13 \%$ of patients a chest film was not obtained within 48 hours of ordering the CTPA. This indicates that in just fewer than half the patients (47\%) a pathological diagnosis was present on the chest film. Clearly the presence of a radiological process on chest X-ray does not rule out the concomitant presence of $\mathrm{PE}$, and we cannot determine what the ordering physician was considering. However the combination of diminishing yield of the CTPA from 2000 to 2010, the younger and healthier patients on whom CTPA is being performed, the underutilization of pre-tests probability and D-dimer, the presence of known lung pathology on a chest film, the drop in V/Q scans not matched by the increase in the number of CTPAs, all attest to the unjustified overuse of CTPA.

Accepted recommendations for the diagnosis of $\mathrm{PE}$ continue to emphasize pre-test probability to guide the

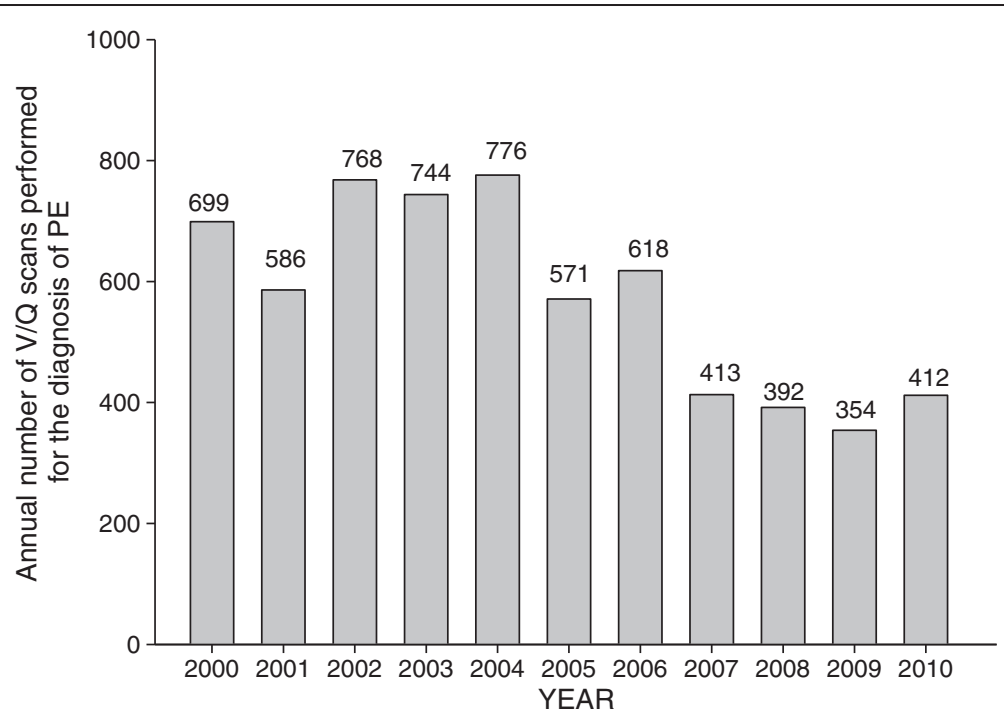

Figure 3 Number of ventilation-perfusion scans (V/Q) performed for the diagnosis of PE from 2000 to 2012. 
Table 5 Trend in number of CTPAs and V/Q scans performed, as well as ED visits and hospital admissions for the years 2000, 2005 and 2008

\begin{tabular}{|c|c|c|c|c|c|}
\hline & 2000 & 2005 & 2008 & $\begin{array}{l}\% \text { change } \\
2000-2005\end{array}$ & $\begin{array}{l}\% \text { change } \\
2005-2008\end{array}$ \\
\hline CTPA & 84 & 1114 & 1883 & 1226 & 69 \\
\hline V/Q scan & 699 & 571 & 392 & -18 & -31 \\
\hline ED visits & 41,464 & 41,004 & 48,813 & -1 & 19 \\
\hline $\begin{array}{l}\text { Hospital } \\
\text { admissions }\end{array}$ & 42,483 & 41,398 & 45,770 & -3 & 10 \\
\hline
\end{tabular}

choice of testing, and the positive predictive value of CTPA remains dependent on the pre-test probability of PE [3-5]. We used documentation of pre-test probability in patients' medical records as a surrogate for assignment of pre-test probability by any means, subjective or objective. It is possible that assessment of pre-test probability was performed by physicians prior to CTPA, but not specifically recorded. However, if this were the case, such assessment of pre-test probability occurred in a rather small minority of patients for the following two reasons. First, D-dimer was only measured in $20 \%$ of all patients with a low or intermediate pre-test probability of PE. Second, only 1 in 5 patients in the high pre-test probability category received anticoagulation while awaiting confirmation of PE with a CTPA. Our study was designed to investigate the rates and indications for the use of CTPA in clinical practice and therefore only includes patients undergoing CTPA. It is possible that patients presented with low risk for PE, were appropriately screened and did not undergo CTPA. We believe that such number is small as our data reveal that younger patients with fewer risk factors for PE were undergoing CTPA.

We assigned pre-test probability retrospectively using the RGS that tends to classify the great majority of patients in the low or intermediate categories and this could limit the interpretability of our findings. However, the limitation of the RGS notwithstanding, it is suited for retrospective calculation and variables included in the RGS can be accurately assessed by systematic chart review (25).

The British Thoracic Society's guidelines advise that should a PE be suspected, the patient should be fully evaluated by an experienced middle-grade doctor (the equivalent of a resident in their third year of training) so that alternative diagnoses are considered and clinical probability for PE is documented. Such practice should then yield a $25 \%$ incidence of PE when CTPA is performed [7]. Our data show that CTPA yields a positive diagnosis in only $11 \%$ to $15 \%$ of all CTPAs. Though subjective assessment by experienced physicians has been shown to be non-inferior to objective assignment of pre- test probability, unfortunately, the present trend in ordering CTPA could make it unlikely for such expertise in the clinical diagnosis of PE to develop. Not only are recommendations not being followed in routine clinical practice, but a greater proportion of CTPA are being performed in patients with lower pre-test probability and on younger and seemingly healthier patients. As guidelines outline how to best utilize CTPAs [3-5,14] and articles lament the over use of CTPAs [8,17-21], Glaser and colleagues demonstrated that a simpler reporting strategy for V/Q scans (PE present, PE absent and non-diagnostic) can be safely implemented, facilitates clearer communication with referring clinicians and may reduce the number of CTPAs ordered [22].

It is possible that the results of this study are isolated to our medical center; however, this is highly suspect. There is little reason to indicate that practice at our center would differ from national practice, especially upon reviewing the literature. Other studies, albeit smaller and covering a shorter time span, are consistent with a dramatic increase in CTPA use [6,7]. Yin and colleagues [23] also found that D-dimer assay was not being used appropriately in a one year evaluation of CTPAs performed in their institution. The increase in CT utilization by our ED is by no means unique; indeed the overall use of CT scan in the ED had risen by $14 \%$ a year since 1995 and by 330\% from 1996 to 2007 [18,24].

\section{Conclusion}

In conclusion, our data indicate that search for an alternate radiologic diagnosis does not justify what appears to be an indiscriminate use of CTPA. CTPA is being increasingly used as the first and only diagnostic test for suspected PE and the frequency of positive CTPAs has declined significantly over time, with younger and healthier patients being tested.

\section{Competing interest}

The authors declare that they have no conflict of interests relating to this work.

\section{Authors' contributions}

SC participated in study design, data collection and entry and drafted the manuscript. PS participated in data collection and entry and contributed to study design. DC performed the statistical analysis and contributed to the manuscript. NG participated in data collection and entry. RC conceived of the study, and participated in its design and coordination and contributed to the manuscript. All authors read and approved the final manuscript.

\section{Author details}

'Division of Pulmonary, Critical Care and Sleep Medicine, The Long Island Jewish Medical Center, The Hofstra North Shore-LIJ School of Medicine, New Hyde Park, New York, NY 11040, USA. ²Division of Pulmonary, Sleep and Critical Care Medicine, University of Pittsburgh Medical Center, Pittsburgh, PA 15213, USA.

Received: 9 July 2012 Accepted: 30 January 2013

Published: 7 February 2013 


\section{References}

1. Gosalia R, Drachman D, Gridley D, Zamora JG, Mamlouk MD, VanSonnenberg E, et al: Pulmonary Embolism at CT Angiography: Implications for Appropriateness, Cost, and Radiation Exposure in 2003 Patients. Radiology 2010, 256:625-32.

2. Mettler FA Jr, Thomadsen BR, Bhargavan M, Gilley DB, Gray JE, Lipoti JA, et al: Medical radiation exposure in the U.S. in 2006: preliminary results. Health Phys 2008, 95:502-7.

3. Stein PD, Woodard PK, Weg JG, Wakefield TW, Tapson VF, Sostman HD, et al: Diagnostic Pathways in Acute Pulmonary Embolism: Recommendations of The PIOPED II Investigators. Am J Med 2006, 119:1048-55.

4. Stein PD, Woodard PK, Weg JG, Wakefield TW, Tapson VF, Sostman HD, et al: Diagnostic Pathways in Acute Pulmonary Embolism: Recommendations of the PIOPED II Investigators1. Radiology 2007, 242:15-21.

5. Moores LK, King CS, Holley AB: Current Approach to the Diagnosis of Acute Nonmassive Pulmonary Embolism. Chest 2011, 140:509-18.

6. Donohoo JH, Mayo-Smith WW, Pezzullo JA, Egglin T: Utilization patterns and diagnostic yield of 3421 consecutive multidetector row CTPA in a busy emergency department. J Comput Assist Tomogr 2008, 32:421-5.

7. Sarojini D, Beddy P, Babar J, Devaraj A: Evolution of CT pulmonary angiography: referral patterns and diagnostic yield in 2009 compared with 2006. Acta Radiol 2012, 53:39-43.

8. Pistolesi M, Pulmonary $\mathrm{CT}$ : Angiography in Patients Suspected of Having Pulmonary Embolism: Case Finding or Screening Procedure? Radiology 2010, 256:334-7.

9. Hall WB, Truitt SG, Scheunemann LP, Shah SA, Rivera MP, Parker LA, Carson SS: The prevalence of clinically relevant incidental findings on chest computed tomographic angiograms ordered to diagnose pulmonary embolism. Arch Intern Med 2009, 169:1961-5.

10. Tanaka N, Matsumoto T, Miura G, et al: Air trapping at CT: high prevalence in asymptomatic subjects with normal pulmonary function. Radiology 2003, 227:776-85.

11. Richman PB, Courtney DM, Friese J, et al: Prevalence and significance of nonthromboembolic findings on chest computed tomography angiography performed to rule out pulmonary embolism: a multicenter study of 1,025 emergency department patients. Acad Emerg Med 2004, 11:642-7.

12. Tresoldi S, Kim YH, Baker SP, et al: MDCT of 220 consecutive patients with suspected acute pulmonary embolism: incidence of pulmonary embolism and of other acute or non-acute thoracic findings. Radiol Med 2008, 113:373-84.

13. Schertler T, Frauenfelder $T$, Stolzmann $P$, et al: Triple rule-out $C T$ in patients with suspicion of acute pulmonary embolism: findings and accuracy. Acad Radiol 2009, 16:708-17.

14. Bounameaux H: Contemporary management of pulmonary embolism: the answers to ten questions. J Intern Med 2010, 268:218-31.

15. Le Gal G, Righini M, Roy P-M, Sanchez O, Aujesky D, Bounameaux H, et al: Prediction of Pulmonary Embolism in the Emergency Department: The Revised Geneva Score. Annals of Internal Medicine. 2006;144:165171.16. Hurwitz LM, Reiman RE, Yoshizumi TT, Goodman PC, Toncheva G, Nguyen G, et al. Radiation Dose from Contemporary Cardiothoracic Multidetector CT Protocols with an Anthropomorphic Female Phantom: Implications for Cancer Induction1. Radiology 2007, 245:742-50.

16. Schattner A: Computed tomographic pulmonary angiography to diagnose acute pulmonary embolism. The good, the bad, and the ugly. Arch Intern Med 2009, 169:1966-1968.

17. Wears RL: The Hunting of the Snark. Ann Emerg Med 2011, 58:465-467.

18. Weir ID, Drescher F, Cousin D, Fraser ET, Lee R, Berman L, Strauss E, et al: Trends in use and yield of chest computed tomography with angiography for diagnosis of pulmonary embolism in a Connecticut hospital emergency department. Conn Med 2010, 74(1):5-9.

19. Kline JA, Courtney DM, Beam DM, King MC, Steuerwald M: Incidence and predictors of repeated computed tomographic pulmonary angiography in emergency department patients. Ann Emerg Med 2009, 54(1):41-8.

20. Costantino MM, Randall G, Gosselin M, Brandt M, Spinning K, Vegas CD: $\mathrm{CT}$ angiography in the evaluation of acute pulmonary embolus. Am J Roentgenol 2008, 191(2):471-4.

21. Glaser JE, Chamarthy M, Haramati LB, Esses D, Freeman LM: Successful and safe implementation of a trinary interpretation and reporting strategy for V/Q lung scintigraphy. J Nucl Med 2011, 52(10):1508-12.
22. Yin F, Wilson T, Della Fave A, Larsen M, Yoon J, Nugusie B, Freeland H, Chow RD: Inappropriate use of D-dimer assay and pulmonary CT angiography in the evaluation of suspected acute pulmonary embolism. Am J Med Qual 2012, 27:74-9.

23. Kocher KE, Meurer WJ, Fazel R, Scott PA, Krumholz HM, Nallamothu BK: National trends in use of computed tomography in the emergency department. Ann Emerg Med 2011, 58:452-62.

24. Wong DD, Ramaseshan G, Mendelson RM: Comparison of the Wells and revised Geneva scores for the diagnosis of pulmonary embolism: an Australian experience. Int Med J 2010, 41(3):258-63.

doi:10.1186/1471-2466-13-9

Cite this article as: Chandra et al:: Finding an alternative diagnosis does not justify increased use of CT-pulmonary angiography. BMC Pulmonary Medicine 2013 13:9.

\section{Submit your next manuscript to BioMed Central and take full advantage of:}

- Convenient online submission

- Thorough peer review

- No space constraints or color figure charges

- Immediate publication on acceptance

- Inclusion in PubMed, CAS, Scopus and Google Scholar

- Research which is freely available for redistribution 